\title{
Solving second order delay differential equations by direct two and three point one-step block method
}

\begin{abstract}
In this paper we present a two point and three point one-step block method for solving second order delay differential equations (DDEs). The one-step block method will solve directly the second order DDEs without reducing to first order equations. The two point and three point one-step block method will compute the solutions for the DDEs at two and three points simultaneously along the interval. These methods will solve the retarded type of DDE of single delay using constant step size. The P- stability and Q-stability are also discussed. The numerical results are presented to illustrate the performance of those block method for solving delay differential equations.
\end{abstract}

Keyword: Delay differential equations; Two point block; Direct method. 